\title{
Aberrant left coronary artery arising from the right sinus of Valsalva: Case reports of a rare entity
}

\author{
Marie-Sophie Kupper, MD, ${ }^{\mathrm{a}}$ Noella Bethuyne, MD, ${ }^{\mathrm{b}}$ Jean Rubay, MD, ${ }^{\mathrm{b}}$ Francois Verhelst, $\mathrm{MD},{ }^{\mathrm{b}}$ Catherine Barrea, MD, \\ and Stéphane Moniotte, MD, PhD, ${ }^{a}$ Brussels, Belgium
}

Supplemental material is available online.

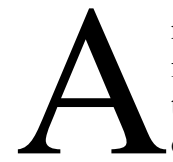

mong the clinically relevant congenital coronary malformations, origin from the wrong aortic sinus seems to be the most common in young trained athletes who die suddenly. The reported incidence is $0.92 \%$ for anomalous origin of the right coronary artery (RCA) from the left sinus and $0.15 \%$ for the left coronary artery (LCA) from the right sinus. ${ }^{1}$ However, these data likely underestimate the true incidence of these abnormalities because these angiographic studies were presumably performed for other clinical indications. In a recent study of 6.3 million US Army recruits, Eckart and colleagues ${ }^{2}$ reviewed the medical records of 277 nontraumatic deaths and found that $34 \%$ were the result of coronary anomalies. No coronary anomaly other than anomalous origin of the LCA from the right sinus of Valsalva was associated with cardiac death.

\section{Clinical Summary}

PATIENT 1. A previously healthy 2-year-old girl presented for evaluation of a murmur. Physical examination revealed stable vital signs, normal heart sounds, and a continuous murmur at the left sternal border. Electrocardiographic, radiographic, and laboratory findings were normal. Transthoracic echocardiography showed a patent ductus arteriosus, and an aberrant LCA from the right sinus of Valsalva was suspected.

Cardiac catheterization demonstrated a single coronary ostium from the right sinus of Valsalva, with the left main coronary artery (LMCA) coursing between the aortic root and the main pulmonary artery (Figure 1). Because of the inherent risk of sudden death associated with this malformation, surgery was proposed. The LMCA was found to be extramural, precluding coronary unroofing. The patent ductus arteriosus was clipped, and given the small coronary artery diameter and single coronary ostium, a coronary bypass oper-

From the Departments of Pediatric Cardiology ${ }^{\mathrm{a}}$ and Cardiac Surgery, ${ }^{\mathrm{b}}$ Cliniques Universitaires St Luc, Université Catholique de Louvain, Brussels, Belgium.

Received for publication Oct 8, 2007; accepted for publication Feb 1, 2008.

Address for reprints: Stéphane Moniotte, MD, PhD, Department of Pediatric Cardiology, Université Catholique de Louvain, 10 avenue Hippocrate, B1200 Brussels, Belgium (E-mail: stephane.moniotte@uclouvain.be).

J Thorac Cardiovasc Surg 2008;136:788-9

$0022-5223 / \$ 34.00$

Copyright $\odot 2008$ by The American Association for Thoracic Surgery doi:10.1016/j.jtcvs.2008.02.007 ation was proposed at a later stage. At the 1-year follow-up, the patient was asymptomatic.

PATIENT 2. A 13-year-old patient was referred for evaluation of multiple syncopes and chest pain associated with exercise. Laboratory findings and electrocardiograms were consistent with myocardial ischemia, and the echocardiogram revealed an aberrant LMCA from the right sinus of Valsalva with significant regional wall motion abnormalities (Figure E1, videoclip).

The LMCA had an intramural course between the aorta and the main pulmonary artery, as confirmed by magnetic resonance coronary angiography (Figure 2). Unroofing of the intramural segment of the LMCA was performed. The unobstructed origin of the LMCA was demonstrated by coronarography 1 year after its reimplantation. At the 5-year follow-up, the patient was free of symptoms (Figure E2).

\section{Discussion}

With the sophistication of noninvasive imaging techniques, the number of coronary artery anomalies identified is increasing. In a recent prospective series of 1950 patients studied by angiography, Angelini ${ }^{1}$ reported an incidence of $5.64 \%$ of coronary anomalies, with an LMCA arising from the right sinus in only $0.15 \%$ of the cases.

Although sudden death was frequently the first symptom, premonitory manifestations occurred in a substantial proportion of individuals with wrong sinus coronary artery origin. Basso and colleagues $^{3}$ reported syncope or chest pain in 7 of 27 athletes within 24 months of sudden death. These symptoms were limited to anomalous LMCA origin, whereas anomalous origin of the RCA proved to be clinically silent. Other authors have reported patients with anomalous RCA who experienced angina, arrhythmia, or syncope before death. ${ }^{4,5}$

These observations emphasize the need to raise the index of clinical suspicion to identify wrong aortic sinus coronary artery origins in young, physically active individuals. They should systematically be excluded from participation in competitive sports until their specific coronary anatomy is delineated. A specific subgroup of patients have an intramural course of the ectopic coronary artery, often entailing a worse prognosis and requiring more aggressive treatment. $^{5,6}$

The current approaches available for repair are 1) the reimplantation of the LMCA in the left sinus of Valsalva; 2) a coronary bypass procedure; and 3) an unroofing procedure resulting in relocation of the coronary artery into the appropriate sinus of Valsalva, with modified orifice shape and full patency after reconstruction.

The surgical approach may also vary depending on the patient's age and specific LMCA anatomy. At the Universite Catholique de Louvain, the favored surgical technique is based on the specific anatomy of the LMCA (presence or absence of an intramural course between the ascending aorta and the main pulmonary artery) and the patient's age and symptoms (Table E1). 

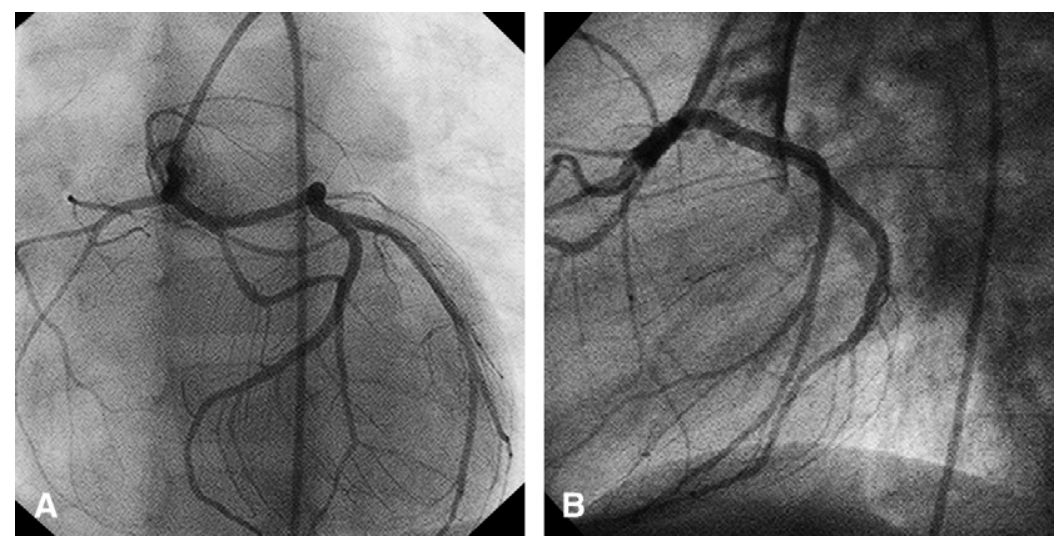

Figure 1. Coronary angiography of patient 1. A, The anteroposterior projection shows an unusual long-segment LMCA. B, The lateral projection shows an anterior origin of the LMCA, with a long segment coursing posteriorly.
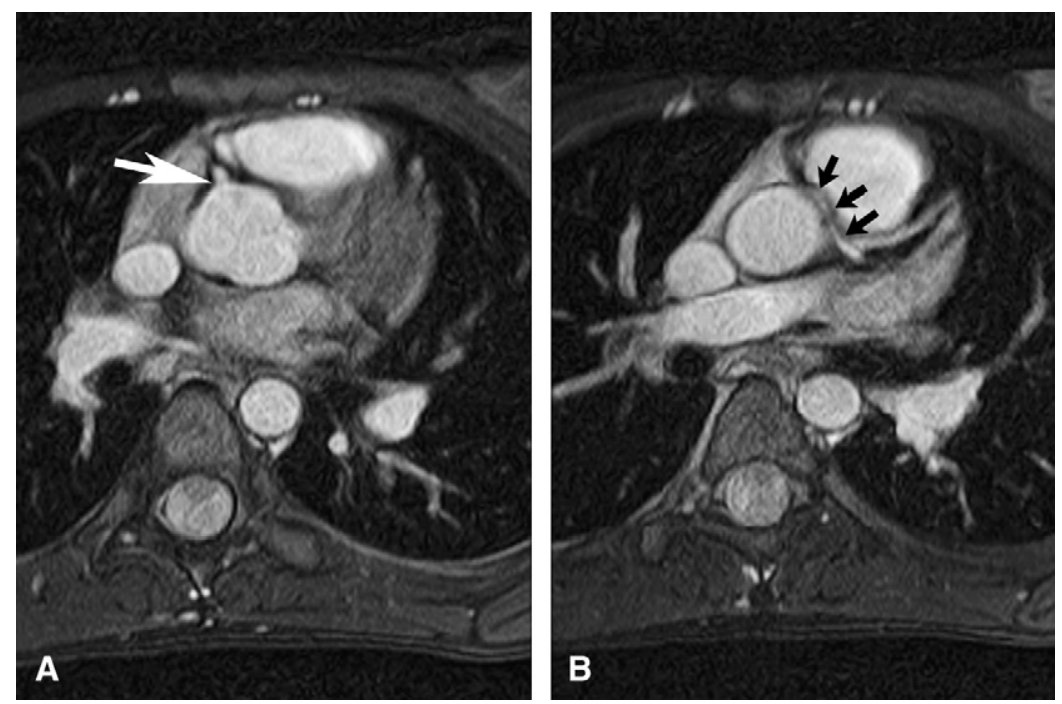

Figure 2. Preoperative magnetic resonance imaging evaluation of patient 2 with 3-dimensional free-breathing coronary magnetic resonance angiography. $A$, The RCA originates from the right sinus of Valsalva (white arrow). B, The interarterial and intramural course of the LMCA originating from the right sinus of Valsalva is clearly visible on the axial images (black arrows).

Although further multicentric studies are necessary to design the optimal management algorithm in patients with this rare entity, the unroofing procedure is preferred in the presence of an intramural segment. ${ }^{7}$ For technical reasons, surgery will be proposed later in life in young asymptomatic patients with an extramural course. Although surgical correction is warranted regardless of the presence of symptoms in the pediatric population, the optimal management of asymptomatic adults is still a matter of debate. Patients aged more than 30 years should probably undergo corrective surgery exclusively in the presence of symptoms.

\section{References}

1. Angelini P. Coronary artery anomalies: an entity in search of an identity. Circulation. 2007;115:1296-305.
2. Eckart RE, Jones SO, Shry EA, et al. Sudden death associated with anomalous coronary origin and obstructive coronary disease in the young. Cardiol Rev. 2006;14:161-3.

3. Basso C, Maron BJ, Corrado D, et al. Clinical profile of congenital coronary artery anomalies with origin from the wrong aortic sinus leading to sudden death in young competitive athletes. J Am Coll Cardiol. 2000;35: 1493-501.

4. Roberts WC, Siegel RJ, Zipes DP. Origin of the right coronary artery from the left sinus of valsalva and its functional consequences: analysis of 10 necropsy patients. Am J Cardiol. 1982;49:863-8.

5. Moustafa SE, Zehr K, Mookadam M, et al. Anomalous interarterial left coronary artery: an evidence based systematic overview. Int $J$ Cardiol. 2007 Aug 13 [Epub ahead of print].

6. Angelini P. Coronary artery anomalies-current clinical issues: definitions, classification, incidence, clinical relevance, and treatment guidelines. Tex Heart Inst J. 2002;29:271-8.

7. Romp RL, Herlong JR, Landolfo CK, et al. Outcome of unroofing procedure for repair of anomalous aortic origin of left or right coronary artery. Ann Thorac Surg. 2003;76:589-95. 

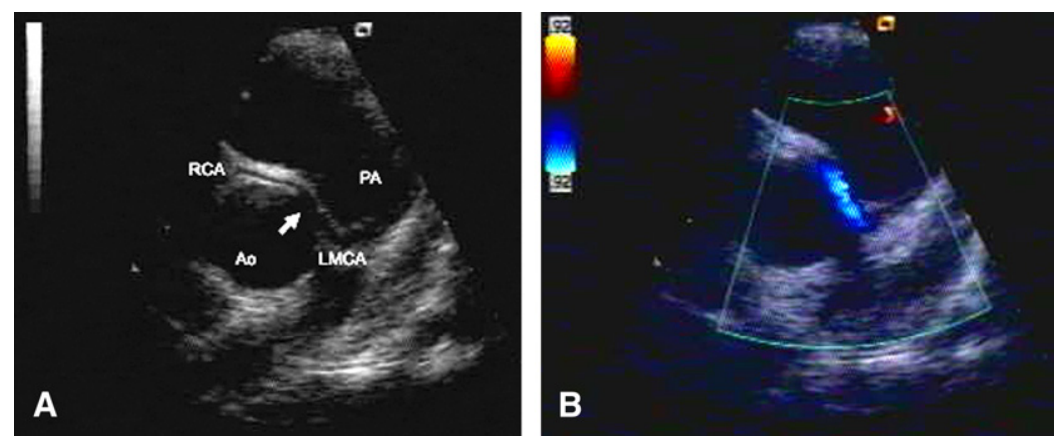

Figure E1. A, Two-dimensional echocardiographic image of an anomalous LMCA (patient 2). There is a clear intramural segment with a common orifice (arrow) and an RCA originating from the right sinus of Valsalva. B, Abnormal direction of blood flow is shown by color Doppler (in blue) in the intramural segment of the LMCA. PA, Pulmonary artery; Ao, ascending aorta. 

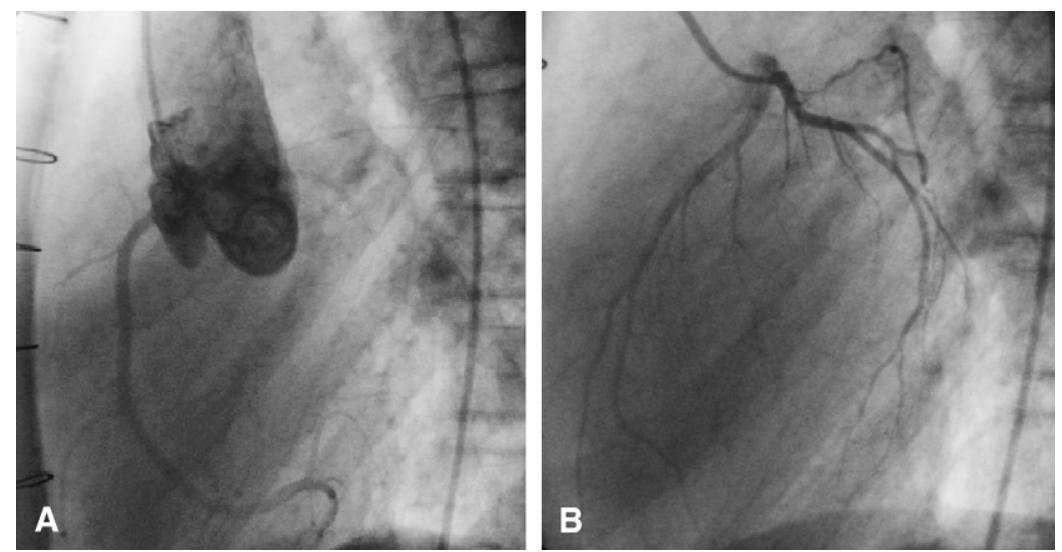

Figure E2. Coronary angiography 1 year after LMCA reimplantation in the left sinus of Valsalva (patient 2). A, The RCA is unobstructed. B, The LMCA originates from the left aortic sinus and gives rise to the left anterior descending and circumflex coronary arteries. 
TABLE E1. Suggested surgical approach based on the patient's age, symptoms, and aberrant left main coronary artery anatomy

Aberrant LMCA from the right sinus of Valsalva

\begin{tabular}{lll} 
& \multicolumn{2}{c}{ Aberrant LMCA from the right sinus of Valsalva } \\
\cline { 3 - 3 } & \multicolumn{1}{c}{ Intramural } & \multicolumn{1}{c}{ Extramural } \\
\hline $0-8 y$ & & \\
Symptomatic & Unroofing & Reimplantation or bypass \\
Asymptomatic & Delayed intervention or unroofing & Delayed intervention \\
$8-35 y$ & & \\
Symptomatic & Unroofing & Reimplantation or bypass \\
Asymptomatic & Unroofing & Reimplantation or bypass \\
$>35 y$ & Unroofing & Reimplantation or bypass \\
Symptomatic & No intervention & No intervention \\
Asymptomatic & & \\
\hline
\end{tabular}

$L M C A$, Left main coronary artery. 